\title{
A unified approach for the fading statistics of heterogeneous compound channels in transparent relay transmissions
}

\author{
Cibile K Kanjirathumkal $^{\dagger^{*}}$, Sameer S Mohammed ${ }^{\dagger}$ and Lillykutty Jacob ${ }^{\dagger}$
}

\begin{abstract}
In this paper, we address the challenging issue of multi-hop cooperative relaying over heterogeneous compound channels, where constituent channels can incorporate topographical variations and respective distribution changes (due to environmental factors). Existing methods in the literature address various issues pertinent to receiver design by assuming a homogeneous framework. Since real-time signal level monitoring at the relay node is impractical in transparent relaying, statistical knowledge of the end-to-end channel is critical for an energy-efficient system design. As the received signal quality and hence the signal-to-noise ratio (SNR) depend on the channel condition, an exact characterisation of the end-to-end compound channel is essential for the implementation of transparent relay networks in a particular coverage area. This demands a realistic heterogeneous compound channel model, having distinct component distributions, that can accommodate variations in per-hop channel distribution. Exact probability density function and cumulative distribution function of the end-to-end compound channel are derived in closed form, through an inverse Mellin transform approach. As there can be many combinations of heterogeneous channels, we propose a unified approach for deriving statistical properties of interest. Two typical cases of heterogeneous channels, Nakagami $\times$ Weibull and Rayleigh $\times$ Weibull, are considered to validate the proposed generic analytical approach. Based on the derived exact statistics of these models, performance metrics, such as coefficient of variation, average SNR, outage probability, and average SER, are evaluated. Through appropriate use of these metrics, optimal power boosting at the relays and quality stream selection for arbitrary diversity combiner units can be done. The pertinent analytical results are also validated through simulation studies to demonstrate the accuracy and applications of the results.
\end{abstract}

Keywords: Mellin Transform; Multi-hop non-regenerative relay; Compound channels; Weibull distribution; Rayleigh distribution; Nakagami-m distribution

\section{Introduction}

With the possibility of providing enhanced network coverage as well as spatial diversity, wireless cooperative relaying is undoubtedly one of the most significant communication strategies today. Of the many possible schemes of relaying, transparent or amplify and forward (AF) relaying is usually preferred, as the processing burden on the relay is minimal, though the compounding channel effects due to cascaded channel gain multiplication is a serious

\footnotetext{
*Correspondence: petercibil@gmail.com

${ }^{\dagger}$ Equal contributors

Department of Electronics and Communication, National Institute of Technology Calicut, Calicut 673601, India
}

issue. However, this demands sophisticated implementation techniques, as it can bring much more attenuation and uncertainty to the transmitted signal than other schemes do. Technologically, the extensive applications of wireless relay transmissions are impedimented by the undesirable features of the wireless transmission environments. One of the fundamental challenges to be addressed for wireless communication is the random fluctuation of the transmitted signal, which is mainly due to the multi-path effects and shadowing. In the context of multihop transparent relaying, additional impairments on the end-to-end channel from multiple relaying, with distinct per-hop distributions due to distinct environments, require special attention as it affects the system design and

\section{是 Springer}

(c) 2013 Kanjirathumkal et al: licensee Springer. This is an Open Access article distributed under the terms of the Creative

Commons Attribution License (http://creativecommons.org/licenses/by/2.0), which permits unrestricted use, distribution, and reproduction in any medium, provided the original work is properly cited. 
receiver performance. Appropriate power amplification by the intermediate relays is another major issue that has to be addressed in AF relay system. Amplification by the relay node based on respective signal strength measurement is impractical in AF systems, as this may increase overall delay and processing burden at the relay node. Hence, a statistical knowledge of the end-to-end channel under consideration is critical, to implement a robust communication system. The main objective of the proposed work was to provide a unified approach to extract the statistical properties of any compound channel, which will enable us to design links that account for per-hop channel distributions and to reap the benefits of diversity.

To address the issue of modeling random fluctuations of the transmitted signal, terrain dependent fading models for direct link transmissions, based on empirical study of the received signal, are available. Extending this to multihop scenario results in homogeneous compound channel models as in [1-3], and is more suitable for indoor applications. The double Rayleigh model analysis using Meijer G-function for multiple-input multiple-output (MIMO) systems in $[4,5]$ is often used in multi-hop relay transmissions where homogeneous product channel effects are experienced. As the relays are physically apart in multihop transmissions, spatial correlation can be neglected in modeling.

In outdoor multi-hop transmissions, the assumption of homogeneous scattering environment for all the constituent links is definitely an approximation; because in actual scenario, cascaded links are often characterised by heterogeneous environment due to scattering density variations. Hence, a more realistic channel model suitable for transparent relay transmissions is presented. The analysis is extended to it so as to make realistic prediction of signal variations at the receiver, and to facilitate appropriate detection strategies. Two cases of heterogeneous channel models, Nakagami $\times$ Weibull $(\mathrm{NW})$ and Rayleigh $\times$ Weibull $(\mathrm{RW})$, are considered to demonstrate the validity of the proposed generic approach. (Nakagami$m$ and Weibull distributions belong to different classes of distributions, while Rayleigh and Weibull belong to same class of distributions.) A homogeneous compound channel model Weibull $\times$ Weibull $($ WW $)$ is also considered for reference and comparison. Appropriate performance metrics required for a transparent relay system are derived for the given compound channel models.

The rest of the paper is organized as follows. A brief discussion on the methodology adopted for analysis, metrics chosen for evaluation, and contributions are presented in the rest of this introduction. In Section 2, closely related work from the literature are discussed. In Section 3 , the cooperative communication system and the channel model under consideration are presented. Exact expressions for the primary statistics like $p d f$ and $c d f$ are derived in Section 4. Performance measures such as coefficient of variation, average SNR, and outage probability are computed in Section 5. Using simulation studies, validation of analytical results, determination of average BER performance of a transmission system, and study of quantilequantile (q-q) profiles for different channel models are done in Section 6. Discussion on the results are included in Section 7, and the paper is concluded in Section 8.

\subsection{Summary of methodology, metrics, and contributions} Instead of exploring new distributions for the end-to-end compound channels, a closer match to the actual scenario can be obtained if product combinations of known distributions are selected according to the topographical variations. This results in distributions that show accurate fit to the practical scenario. Analytical study of this unexplored framework for the compound channels in multihop AF relay communication is too complex and often uses approximate solutions. The generic approach that we propose can be used to obtain the exact probability density functions $(p d f s)$ of new random variables (RVs) that are algebraic combinations or functions of independent RVs.

Moment generating function $(m g f)$ and Mellin transform (MT) are the two commonly used tools for the computation of distribution moments [6,7] in communication theory. As we focus on a generic approach for finding the $p d f$ of a heterogeneous compound distribution, the product convolution property of MT for independent RVs is exploited. Also, as MT translates exponentials to polynomials, complexity of the analysis can thus be reduced to a certain extent. Using certain other properties of MT, convenient computation of signal-to-noise ratio (SNR) statistics is also possible. The ease of computation of metrics related to SNR, convenient extension to three or more hop counts, and computation of the end-to-end moments without the knowledge of overall $p d f$ are some of the added benefits of this transform.

We can find the $p d f$ from the inverse transform. The inverse transform computation requires the evaluation of a contour integral. For the case of known standard homogeneous compound distributions, this integral evaluation can be done directly by using the Meijer G-function which is available as built-in functions in computing software packages [8]. But it is not readily available for every product distributions of interest. Therefore, conversion of the contour integral to appropriate Meijer-G format is essential for the evaluation. However, the coefficients of the transform variable may not be identical for two different distributions. This issue is solved in our work by applying gamma duplication formula and reduction rule to one of the component distributions.

Modeling the distribution of the signal fluctuation is just the first step. In order to employ a fading model in link 
budget, it is also vital to determine suitable performance metrics based on the statistical properties of the model. A performance metric involving higher order moments is often required to asses the possible signal variations. Hence, a metric called coefficient of variation $(\mathrm{CV})$ is derived which reflects the slightest change in distribution with respect to the fading factors and hop counts. Performance prediction adaptive to hop count and terrain is essential for robust system implementation. Hence, outage probability is evaluated for different channel models using the exact $p d f$ of SNR distributions.

As power allocation in AF system critically depends on the SNR and power constraints of relays, accurate estimation of average SNR, which is sensitive to hop count and individual hop distributions, is essential for the effective implementation of the system. Hence, an expression for the average SNR is derived (in terms of relay amplification factor). It is then used to simulate a two-hop AF relay transmission system and the bit error performance is analysed for different compound channel models. Metrics like CV and average SNR derived can be used to select appropriate branches in a selection or switched diversity combining systems $[9,10]$.

\section{Related works}

Most of the previous works on product $p d f$ are based on $m g f$. When known standard distributions with the available $m g f$ are used for the $p d f$ and other statistics, readily available results from computing software packages can be used for evaluation.

In some of the previous works $[6,8,11]$, harmonic distribution is assumed for the end-to-end SNR. But this is based on the condition that the real-time channel state information (CSI) is available at the relay node so that the relay amplification factor can be chosen accordingly. However, real-time CSI at relay node is impractical for the case of a transparent relay networks. In such situations, the end-to-end SNR distribution is to be obtained from the product distribution of the compound channel coefficients as we show in Section 3. Based on the upper bound obtained using the inequality between harmonic and geometric means of positive RVs, the moments of the end-to-end SNR for Rayleigh, Nakagami- $m$, and Nakagami- $n$ fading channels are derived in [6],[8]. The approximation of harmonic distribution is valid only at high SNR, as per the derivation. Same approach based on bounds is followed in $[11,12]$ for Nakagami- $m$ and generalized Gamma channels. The related works with nonidentical constituent distributions either deal with same class of distributions with non-identical scale parameters or resort to approximations.

Exact primary statistics, $p d f$ and $c d f$, for the product of $n$-Rayleigh RVs are derived in [7], using the Meijer G-function. The corresponding infinite series expansions of the expressions from subroutine packages are also provided. Exact expressions for capacity and SNR for $n$-Weibull distributions are derived in [2], where approximations to $p d f$ are also provided. Using inverse Laplace transform of the $m g f$ of product RVs, compound $p d f$ for Nakagami- $m$ distribution is derived in [13] in terms of Hyper geometric functions. Performance measures for a homogeneous $n$-Weibull distribution are derived in [3], where only the statistics based on moments of homogeneous compound channels are considered. The primary statistics like fading distributions and SNR distribution for homogeneous or heterogeneous compound channels are not obtained in this work.

In [11] and [1], Nakagami- $m$ distribution is assumed for the individual channels, whose SNR distribution falls under another known standard distribution, namely Gamma. Since known identical $p d f$ s are used, for the constituent channels, mathematical subroutines can directly provide the corresponding $p d f$ of the compound channel in special function formats. Using the Fox's $\mathrm{H}$-function, the $p d f, c d f$, and $m g f$ for the $\mathrm{N}$-product generalized Nakagami- $m$ distribution for different $m$ are found in [14], along with channel capacity and amount of fade (AoF). However, series form expansions of Fox's $\mathrm{H}$-function are tedious to obtain, especially when multiple order poles are involved. Using $m g f$ approach, approximate average bit error rate and AoF are computed for identical $n$ Weibull distributions in [15]. In this work, the end-to-end SNR is taken as the product distribution of individual SNRs for the AoF computation. The upper bound of SNR in a dual-hop system having non-identical Weibull distributions is considered in [16], where the overall SNR effect is approximated by selecting a channel having the minimum SNR. The same approach is followed in [17], for cascaded Rayleigh and Rican distribution, where effectively, the compound channel reduces to single channel model for further analysis. Despite these contributions, a unified approach for the exact characterisation of compound channels with combinations of distinct component distributions is not available in the literature.

An explicit investigation on the fading effects due to heterogeneous cascading and corresponding metrics evaluation is essential. Certain distributions, considered as special cases of other distributions, can be derived from the original distribution by parameter substitution. But for the integral inversion, when product effect is considered, parameter substitution to the original distribution may not produce accurate results. As the integral will only pick up the values of the residues at each pole, a single contour of integration is required for the inverse computation. This may not be always possible due to the change in the transform variable coefficients of the corresponding function arguments. This issue has to be addressed in 
order to analyse heterogeneous case, and it is in this aspect that our work is different from the above works.

\section{Compound channel and system model}

Our focus is on the exact characterisation of the fading statistics of cascaded links having distinct constituent distribution for each link, which are referred to as heterogeneous compound channels. Formation of such a channel is illustrated in Figure 1.

\subsection{Compound channel model}

We represent the composite channel coefficient by the product of $n$ independent, but not necessarily identically distributed RVs $X_{i}$, i.e.,

$$
V=\prod_{i=1}^{n} X_{i}
$$

where $X_{i} s$ are non negative RVs that can be of any distribution depending on the nature of the propagation environment. This model is justified as we will see in the system model in the following subsection. Weibull and Nakagami- $m$ are the two terrain specific distributions which are commonly used to model the empirical data depicting the environmental variations in outdoor communications. A generic Weibull $p d f$, unlike the basic Weibull $p d f$ considered in $[2,15,16]$, and suitable for radio systems operating in any frequency range is selected as the reference fading distribution:

$$
f_{W_{i}}(w ; \alpha, \beta)=\frac{\beta}{\alpha}\left(\frac{w}{\alpha}\right)^{\beta-1} e^{-\left(\frac{w}{\alpha}\right)^{\beta}} U(w) ; \alpha>0, \beta>0,
$$

where $U(x)$ is the unit step function, and $\alpha$ (resp. $\beta$ ) represents the scale (resp. shape) parameter. Note that, here, the exponentiated RV value ' $w$ ' is raised to a variable power equal to fading factor $(\beta)$. Therefore, this distribution can depict the zonal variations in the topography (as $\beta$ is a terrain dependent factor) more effectively than other distributions. In order to study the effects of heterogeneous compounding, another entirely different distribution namely Nakagami- $m$ with $p d f$

$$
f_{Y_{i}}(y ; \Omega, m)=\frac{2}{\Gamma(m)}\left(\frac{m}{\Omega}\right)^{m} y^{2 m-1} e^{-\left(\frac{m y^{2}}{\Omega}\right)} U(y) ; \Omega>0, m \geq \frac{1}{2},
$$

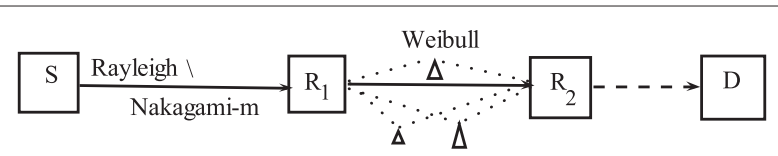

Figure 1 The heterogeneous compound channel formation in a multi-hop transparent relay network. A realistic channel model that accounts for the scattering density variations of the environment is considered for the statistical analysis. is selected along with the Weibull distribution. The parameter $m$ is also known as the fading factor and $\Omega=$ $E\left[Y^{2}\right]$ is the scaling factor. $E[$.$] is the expectation operator.$ In Nakagami- $m$ distribution, the exponentiated squared RV value ' $y$ ' is multiplied by the fading factor $(m)$. Hence, variability of this distribution will be less compared to Weibull distribution.

The RV representing the channel fading in an environment having uniform local scatterers is usually characterised by Rayleigh distribution, given by the $p d f$

$$
f_{R}(r ; \lambda)=2\left(\frac{r}{\lambda^{2}}\right) e^{-\left(\frac{r}{\lambda}\right)^{2}} U(r),
$$

where $\lambda^{2} / 2$ denotes the variance. Note that the exponentiated RV in this case is raised to a constant power of 2 and belongs to the same class of Weibull distribution. Two cases of heterogeneous compound channels formed by the combinations of these distributions are considered to check the validity of the unified approach.

\subsection{System model}

We extend the two-hop system model in [18] to $n$ hops. With reference to Figure 1, there are $n$ hops between source and destination. Using this system model, the signal degradation due to changes in hop count and channel distributions can be assessed. The received signal at destination at any epoch $k$ is represented as

$$
y(k)=v_{e f}(k) d(k)+e_{e f}(k),
$$

where $v_{e f}(k), e_{e f}(k)$, and $d(k)$ are the effective end-to-end channel fading coefficient, effective noise, and the source data, respectively. The effect of end-to-end channel on signal and noise is evident from the given system model. Here,

$$
\begin{aligned}
v_{e f}(k) & =\prod_{j=1}^{n-1} A_{j} \prod_{i=1}^{n} X_{i}(k) \\
e_{e f}(k) & =\sum_{l=1}^{n-1} \prod_{j=l}^{n-1} A_{j} \prod_{i=l+1}^{n} X_{i}(k) e_{l}(k)+e_{n}(k),
\end{aligned}
$$

where $A_{j}$ denotes the amplification factor of the $j^{\text {th }}$ relay, $X_{i}$ denotes the fading coefficient of $i^{\text {th }}$ hop, $e_{l}$ denotes the additive noise at the $l^{\text {th }}$ relay, and $e_{n}$ denotes the additive noise at the destination. As exact CSI is not available at intermediate relay nodes for a non-regenerative system, minimum amplification by the relay is considered as the bound (worst case condition). This imposes the condition that $A_{1}=A_{i}=A_{n-1}=1$, and therefore, effective channel coefficient $v_{e f}=V$ (defined in Equation 1). Under this situation, the relays act just like nodes that establish connectivity to the succeeding nodes. Moreover, considering normalised channel coefficients $X_{i}$ s, the additive noise effect get reduced due to channel multiplication. In short, the signal (data) distortion due to channel attenuation 
(multiplication) becomes much more significant than the additive noise.

\section{Exact primary statistics of compound channels}

In this section, the $p d f$ and $c d f$ of compound channels are derived, and the multiplicative effects of channel coefficients are investigated for homogeneous as well as heterogeneous cases. The approach can be extended to any combinations of distributions like Erlang, Beta, Gamma, etc., for which the MT are defined.

\subsection{Probability density function}

Using MT and its properties, computations can be made simpler for product RVs. The MT of a $p d f f_{X}(x)$ is defined as [19]:

$$
M\left(f_{X}(x), s\right)=\int_{0}^{\infty} x^{s-1} f_{X}(x) d x
$$

and it is denoted by $M_{X}(s)$, where $s=a+j b \in C$ is a complex transform variable. This transform operator represents the second kind characteristic function. The usual techniques of conditioning on $R V \mathrm{~s}$ or Jacobian transformations are no longer required if Mellin transform properties are applied; and the transform of the productpdf can be readily converted to moments of the compound channel $p d f$.

\subsection{1 pdf of $n$-Weibull channel}

The MT for the given $n$-Weibull distribution, using the MT properties [3,19], is

$$
M\left[f_{V}(v), s\right]=\alpha^{(s-1) n} \Gamma^{n}\left(\frac{s+\beta-1}{\beta}\right)=M_{V}(s),
$$

where $V$ represents the product of $n$-Weibull RVs. By setting $s=p+1$, various moments of $n$-hop relay system can be obtained as [3],

$$
m_{p}^{(W)}=E\left[V^{p}\right]=\alpha^{n p} \quad\left[\Gamma\left(1+\frac{p}{\beta}\right)\right]^{n} .
$$

The mean and variance of the $n$-hop relay system can be easily computed from Equation 9. The $p d f$ of $n$-Weibull channel can be derived from the end-to-end moments by finding the inverse of the product transform, defined as

$$
f_{V}(v)=\frac{1}{2 \pi j} \int_{c-j \infty}^{c+j \infty} v^{-s} M\left(f_{V}(v), s\right) d s .
$$

Integration path $\operatorname{Re}(s)=c$ is taken in the fundamental strip of the MT. The inverse MT of Equation 8 yields $n$ Weibull pdf as

$$
f_{V}(v)=\left(\frac{\beta}{\alpha^{n}}\right) \frac{1}{2 \pi j} \int_{L}\left[\Gamma\left(1-\frac{1}{\beta}+s^{\prime}\right)\right]^{n}\left(\frac{v^{\beta}}{\alpha^{n \beta}}\right)^{-s^{\prime}} d s^{\prime}
$$

Note that, to express the integral as a Meijer G-function, it is rewritten as Equation 11 using an appropriate variable substitution $s^{\prime}=\frac{s}{\beta}$. This integral equation can be reduced to the Meijer G-function as:

$$
f_{V}(v)=\frac{\beta}{\alpha^{n}} G_{0, n}^{n, 0}\left(\left.\frac{v^{\beta}}{\alpha^{n \beta}}\right|_{1-\frac{1}{\beta}, 1-\frac{1}{\beta} \ldots} ^{-}\right) .
$$

See Appendix for the definition of general Meijer G-function.

\subsection{2 pdf of heterogeneous channel-Nakagami $\times$ Weibull}

Following the transform development steps in [3], the MT of the Nakagami- $m$ channel can be obtained as

$$
M\left[\left(f_{Y}(y), s\right)\right]=\frac{\Omega}{m}^{\frac{s-1}{2}} \frac{\Gamma\left(m+\frac{s-1}{2}\right)}{\Gamma(m)} .
$$

Considering Weibull as the succeeding constituent channel, the MT of the NW compound $p d f$ (denoted as $\left.f_{\mathrm{NW}}(v)\right)$ can be obtained as

$M\left[\left(f_{\mathrm{NW}}(v), s\right)\right]=\left(\frac{\Omega \alpha^{2}}{m}\right)^{\frac{s-1}{2}} \frac{1}{\Gamma(m)} \Gamma\left(m+\frac{s-1}{2}\right) \Gamma\left(\frac{s+\beta-1}{\beta}\right)$.

Corresponding to this, the $p^{\text {th }}$ moment becomes

$$
m_{p}^{(\mathrm{NW})}=\left(\frac{\Omega \alpha^{2}}{m}\right)^{\frac{P}{2}} \frac{1}{\Gamma(m)} \Gamma\left(m+\frac{p}{2}\right) \Gamma\left(1+\frac{p}{\beta}\right) .
$$

The NW compound $p d f$ is found by inverting the transform given in Equation 14. Even though $\mathrm{H}$-function can be used to represent the inverse integral, as the series form expansion of $\mathrm{H}$-function is often difficult and tedious, most of the computational packages make use of Meijer G-function integral format for the evaluation of definite and indefinite integral equations. By using Gamma reduction rules along with variable transformation, the results can be expressed in Meijer G-function, which makes the computation accurate. Software packages like Mathematica and Matlab can be used for the computation and simplification of integral equations of known standard distributions which are homogeneous. But as these packages do not contain built-in subroutines for such heterogeneous cases, closed-form expressions must be derived in the required format so that accurate computation is possible. Taking the inverse MT of Equation 14, the compound channel $p d f$ of Nakagami $\times$ Weibull distributions can be expressed as

$$
\begin{aligned}
f_{\mathrm{NW}}(v)= & \frac{1}{2 \pi j} \int_{L}\left(\frac{\Omega \alpha^{2}}{m}\right)^{\frac{s-1}{2}} \frac{1}{\Gamma(m)} \Gamma\left(m+\frac{s-1}{2}\right) \\
& \times \Gamma\left(\frac{s+\beta-1}{\beta}\right) v^{-s} d s .
\end{aligned}
$$


In order to translate the variable coefficient in the argument of the last term to unity, set $\frac{s}{\beta}=s^{\prime}$ which results in

$$
\begin{aligned}
f_{\mathrm{NW}}(v)= & \frac{\beta}{\Gamma(m)}\left(\frac{\Omega \alpha}{m}\right)^{\frac{1}{2}} \frac{1}{2 \pi j} \int_{L} \Gamma\left(m-\frac{1}{2}+\frac{\beta s^{\prime}}{2}\right) \\
& \times \Gamma\left(1-\frac{1}{\beta}+s^{\prime}\right)\left(\frac{\Omega \alpha^{2}}{m}\right)^{\frac{\beta s^{\prime}}{2}} v^{-\beta s^{\prime}} d s^{\prime} .
\end{aligned}
$$

Now, application of gamma reduction rule (see Appendix) to this will transform the integral to the format of Meijer G-function. Hence, in terms of Meijer G-function, the pdf reduces to

$$
f_{\mathrm{NW}}(v)=\frac{K \beta b^{b}}{D \Gamma(m)} G_{0, b}^{b+1,0}\left(\left.\frac{v^{\beta}}{\left(b^{\frac{1}{2}} D\right)^{\beta}}\right|_{\frac{2 m-1}{\beta}} ^{-}, \frac{2 m+1}{\beta}, \ldots, \frac{2 m+\beta-3}{\beta}, 1-\frac{1}{\beta}\right),
$$

where $b=\frac{\beta}{2}, D=\left(\frac{\Omega \alpha^{2}}{m}\right)^{\frac{1}{2}}$, and $K=\frac{\sqrt{2 \pi}}{2 \pi^{b / 2}}$. This is computed for specific values of $m, \beta$ and is given in the Appendix.

\subsection{3 pdf of heterogeneous channel-Rayleigh $\times$ Weibull}

The approach can be extended to the RW heterogeneous compound channel. Rayleigh distribution can be considered as a special case of Weibull by using $\beta=2$. The MT of the Rayleigh distribution can be obtained as

$$
M\left[\left(f_{R}(r), s\right)\right]=\lambda^{s-1} \Gamma\left(\frac{s+1}{2}\right) .
$$

The MT of the RW compound $p d f$ (denoted as $f_{R W}(w)$ ) is thus

$$
M\left[\left(f_{\mathrm{RW}}(v), s\right)\right]=\lambda^{s-1} \Gamma\left(\frac{s+1}{2}\right) \alpha^{(s-1)} \Gamma\left(\frac{s+\beta-1}{\beta}\right) .
$$

Corresponding to this, the $p^{\text {th }}$ moment becomes

$$
m_{p}^{(\mathrm{RW})}=\lambda^{p} \Gamma\left(\frac{p+2}{2}\right) \alpha^{(p)} \Gamma\left(\frac{p+\beta}{\beta}\right) .
$$

Using the inverse Mellin transform and denoting $\lambda . \alpha=$ $\alpha_{2}$, the compound channel $p d f$ of Rayleigh $\times$ Weibull distribution can be expressed as

$f_{\mathrm{RW}}(v)=\left(\frac{\beta}{\alpha_{2}}\right) \frac{1}{2 \pi j} \int_{L} \Gamma\left(1-\frac{1}{\beta}+s^{\prime}\right) \Gamma\left(\frac{1}{2}+\frac{\beta s^{\prime}}{2}\right) k^{-s^{\prime}} d s^{\prime}$,

where $k=\left(\frac{v^{\beta}}{\alpha_{2}^{\beta}}\right), s=\beta s^{\prime}$. The application of gamma reduction formula to the second of the two gamma functions will reduce the integrand to

$$
f_{\mathrm{RW}}(v)=\left(\frac{\beta}{\alpha_{2}}\right) K \frac{1}{2 \pi j} \int_{L} \Gamma\left(1-\frac{1}{\beta}+s^{\prime}\right) \Lambda\left(\frac{k}{b}\right)^{-s^{\prime}} d s^{\prime},
$$

where $\Lambda=\prod_{i=0}^{\beta / 2-1} \Gamma\left(\frac{1}{\beta}+\frac{2 i}{\beta}+s^{\prime}\right)$. In terms of Meijer G-function, the heterogeneous compound $p d f$ becomes

$$
f_{\mathrm{RW}}(v)=\left(\frac{\beta}{\alpha_{2}}\right) K G_{0, \frac{\beta}{2}}^{\frac{\beta}{2}+1,0}\left(\left.\frac{k}{b}\right|_{1-\frac{1}{\beta}, \frac{1}{\beta}, \frac{3}{\beta} \ldots, 1-\frac{1}{\beta}} ^{-}\right) .
$$

Corresponding expression for the given specific parameter values is given in the Appendix. The exact expressions for the various compound $p d f s$ are plotted in Figure 2 and will be discussed along with other results in Section 6 .

\subsection{Cumulative distribution function}

Note that the $p d f$ expressions given in Equations 11, 17, and 23 have two variables, the transform variable $s^{\prime}$ and the $p d f$ argument $v$. Integrating the $p d f$ with respect to the variable $v$ to an integration limit of a given threshold value yields the exact $c d f$. The double integral can then be rewritten to express the inner integral in terms of $v$ and the outer integral in terms of $s^{\prime}$. The former one is then subjected to some algebraic manipulations ( by making use of Gamma reduction rule) so that the exact $c d f$ can be expressed in terms of Meijer G-functions.

\subsection{1 cdf of the $n$-Weibull compound channel}

By integrating Equation 11 with respect to the product variable $v$ inside the contour integral, and separating out those terms in $v$ only, we have

$$
T=\int_{0}^{v_{t}} v^{-\beta s^{\prime}} d v=\frac{t v_{t}^{-\beta s^{\prime}}}{\beta\left(\frac{1}{\beta}-s^{\prime}\right)},
$$

where the upper limit of the integration $v_{t}$ represents the threshold value for the product RV of the WW distribution. Using gamma reduction rules, $\left(\frac{1}{\beta}-s\right)$ can be

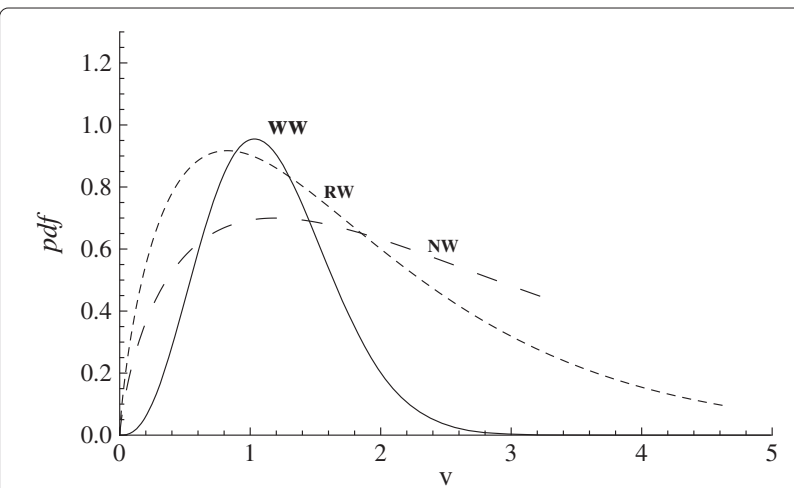

Figure $2 p d f$ comparison plots for Weibull $\times$ Weibull(WW), Rayleigh $\times$ Weibull (RW) as well as Nakagami $x$ Weibull (NW) distributions. Variations in the density function due to homogeneous and heterogeneous channel assumptions are evident from this figure. For comparing the statistical behaviour of the channels, fixed scaling and fading parameters of the distributions are used for plotting the graph $(\alpha=\lambda=\Omega=1, \beta=m=4)$. 
written as $\frac{\Gamma\left(1+\frac{1}{\beta}-s\right)}{\Gamma\left(\frac{1}{\beta}-s\right)}$. Doing some algebraic manipulations and substituting the result to the original integral equation yield the $c d f$ :

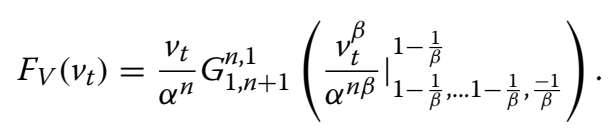

\subsection{2 cdf of Nakagami- $m \times$ Weibull channel}

By integrating Equation 17 with respect to $v$ inside the contour integral, we have

$$
\begin{aligned}
F_{\mathrm{NW}}\left(v_{t}\right)= & \frac{b^{m-1}}{D \Gamma(m)} \frac{K v_{t}}{2 \pi j} \int_{L} \Pi_{i=0}^{b-1} \Gamma\left(\frac{2 m-1}{\beta}+i / b+s^{\prime}\right) \\
& \times \Gamma\left(1-\frac{1}{\beta}+s^{\prime}\right) \frac{\Gamma\left(\frac{1}{\beta}-s^{\prime}\right)}{\Gamma\left(\frac{1}{\beta}-s^{\prime}+1\right)}\left(\frac{v_{t}^{\beta}}{D^{\beta}}\right)^{-s^{\prime}} d s^{\prime} .
\end{aligned}
$$

Evaluation of this integral can be done as described in the previous case, and thus the $c d f$ in terms of the corresponding Meijer G-function is

$$
F_{\mathrm{NW}}\left(v_{t}\right)=\frac{v_{t} b^{m-1}}{D \Gamma(m)} K G_{1, \beta / 2}^{\beta / 2+1,1}\left(\left.\frac{v_{t}^{\beta}}{D^{\beta}}\right|_{\frac{2 m-1}{\beta}, \frac{2 m+1}{\beta}} ^{1-\frac{1}{\beta}} \frac{2 m+3}{\beta} \ldots 1-\frac{1}{\beta}, \frac{-1}{\beta}\right) .
$$

In order to plot the $c d f$, the function may be computed for some specific parameter values, as given in the Appendix.

\subsection{3 cdf of Rayleigh $\times$ Weibull channel}

Following the same procedure as above, the $c d f$ of the RW compound channel can be obtained by integrating Equation 23 with respect to $v$ inside the contour integral, resulting in

$$
F_{\mathrm{RW}}\left(v_{t}\right)=\frac{v_{t}}{\alpha_{2}} K G_{1, \beta / 2}^{\beta / 2+1,1}\left(\left.\frac{v_{2}^{\beta}}{\alpha_{2}^{\beta} b}\right|_{1-\frac{1}{\beta}, \frac{1}{\beta}, \frac{3}{\beta} \ldots 1-\frac{1}{\beta}, \frac{-1}{\beta}} ^{1-\frac{1}{2}}\right) .
$$

Using computation software (Mathematca), exact $c d f$ expressions are plotted in Figure 3, for various channel types, and are discussed in Section 6.

\section{Application of the results-performance evaluation metrics}

As suggested in [20], in the general context of describing the behaviour of a diversity system with arbitrary combining techniques, the channel quality based on channel statistics can be used for performance evaluation. Three different measures have been investigated to evaluate the performance of multi-hop networks using diversity schemes.

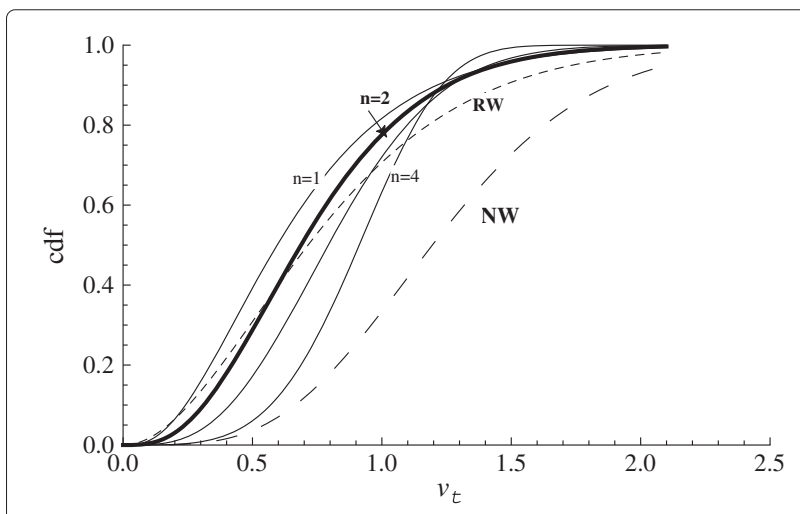

Figure 3 The $c d f$ comparison plots for various hop counts of $n$-Weibull (with $n=1-4$ ) and Rayleigh $\times$ Weibull(WW) as well as Nakagami $x$ Weibull (NW) distributions. The required linear dynamic range of the receiver can be selected based on these plots. Fixed scaling and fading parameters of the distributions $\alpha=\lambda=\Omega=1, \beta=m=4$ are used for plotting the graph.

\subsection{Coefficient of variation-CV}

$\mathrm{CV}$ is a suitable metric for comparison and evaluation of channel (signal) variability due to cascading order and fading distributions. Investigation about the variability of the distribution of the compound channel coefficients is quite useful, as it accounts for the variability to the data being transmitted. In practice, the main problem with variance (v) is that it is expressed in units that are square of the units of observations. Since standard deviation (SD) is having the same unit as mean, it is preferable to use the ratio of SD $(\sigma)$ to mean $(\mu)$. This measure is better than variance as it takes the scale of measurement out of variability considerations. The possible signal deviation due to parameter variations and order of multiplications can be computed using this ratio measure[3]. The first and second order moments required for the evaluation of $\mathrm{CV}$ can be computed using the derived expression for the $p^{\text {th }}$ order moments of the respective compound channels. As per the definition,

$$
\mathrm{CV}=\frac{\sigma}{\mu}=\frac{\sqrt{v}}{\mu} .
$$

In the case of WW channel, using Equation 9 with $n=2$,

$$
\mathrm{CV}^{(\mathrm{WW})}=\sqrt{\frac{\Gamma^{N}(1+2 / \beta)}{\Gamma^{2 N}(1+1 / \beta)}-1 .}
$$

Similarly, using the $p^{\text {th }}$ order moments given by Equation 15, the CV for the NW compound channel can be obtained as

$$
\mathrm{CV}^{(\mathrm{NW})}=\sqrt{\frac{\Gamma(m) \Gamma(m+1) \Gamma\left(1+\frac{2}{\beta}\right)}{\Gamma^{2}(m+1 / 2) \Gamma^{2}(m+1 / \beta)}-1 .}
$$


Using the moments given by Equation 21, the CV for the RW distributed channel can be obtained as

$$
\mathrm{CV}^{(\mathrm{RW})}=\sqrt{\frac{4 \Gamma(1+2 / \beta)}{\pi \Gamma^{2}(1+1 / \beta)}-1 .}
$$

The CV plots for the above mentioned compound channels are shown in Figure 4, in which is also provided the simulation results to demonstrate the accuracy of the derived expressions.

\subsection{Average SNR}

SNR is a commonly used performance measure in communication receivers and is an indicator of the overall fidelity of the system [20]. In the context of a communication system subject to fading impairment, more appropriate performance measure is the average SNR, where the term 'average' refers to statistical averaging over the probability distribution of the SNR. In order to use this measure for the system design, expressions are derived that involve overall relay amplification factor also. The instantaneous received SNR is given by

$$
\gamma=V^{2} \frac{E_{b}}{N_{0}}
$$

where $v$ is the RV representing the compound channel coefficient as specified in Equation $1 . E_{b}$ is the average bit energy and $N_{0}$ is the corresponding additive white Gaussian noise power spectral density of the end-to-end system. The corresponding average SNR is

$$
\bar{\gamma}=E[\gamma]=E\left[\frac{E_{b}}{N_{0}} V^{2}\right] .
$$

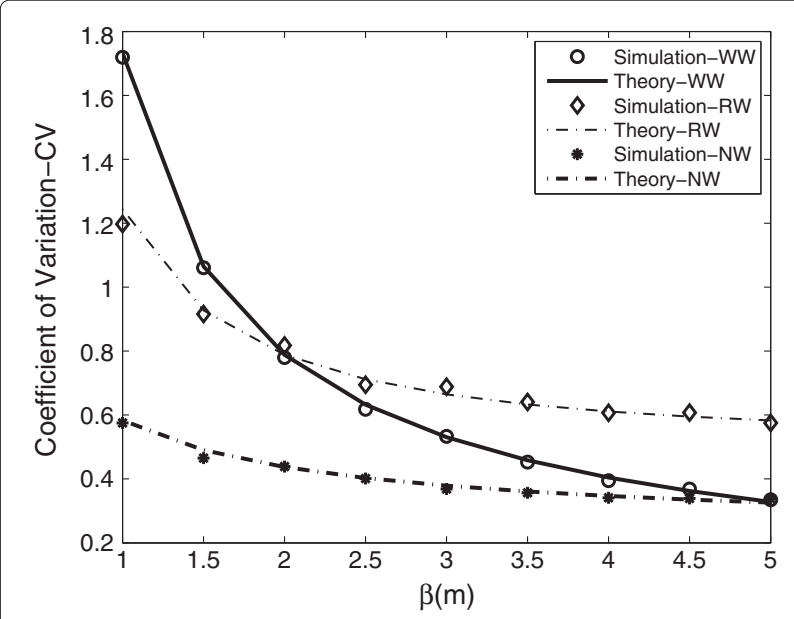

Figure 4 Empirical and theoretical CV plots of Weibull $x$ Weibull (WW), Rayleigh $\times$ Weibull (RW), and Nakagami $\times$ Weibull (NW) distributions. This performance metric accounts for the channel variability and can be selected as a quality index of the channel.
In order to use this statistics in the system design, it is more beneficial to express the overall bit $\mathrm{SNR}, \frac{E_{b}}{N_{0}}$, in terms of per-hop bit SNR, represented by $\frac{e b_{j}}{n 0_{j}}$, and relay amplification factors $A_{j}$. Therefore, using the system model Equations 5 and 6, the overall bit SNR can be written as

$$
\frac{E_{b}}{N_{0}}=E\left[\prod_{j=1}^{n-1} A_{j}^{2} \frac{e b_{j}}{n 0_{j}}\right]
$$

Under the assumption of constant $\frac{e b_{j}}{n 0_{j}}$ that averages to unity in each single hop, $\frac{E_{b}}{N_{0}}$ becomes $A^{2}$, where $A=$ $\prod_{j=1}^{n-1} A_{j}$.

Based on the properties of MT, various moments and the distribution of $V^{2}$ can be computed easily, without the need for $p d f$ transformation approaches followed in $[2,20]$. The proposed approach will be suitable for computing the $p d f$ of SNR of any arbitrary combination of product distributions. Denoting $V^{2}$ by $\Upsilon$, the MT of $\Upsilon$ is given by $M_{\Upsilon}(s)=E\left[V^{2 s-2}\right]=M_{V}(2 s-1)$. The MT of the $p d f$ of $\gamma$ is

$$
M_{\gamma}(s)=E\left[\gamma^{s-1}\right]=\left(A^{2}\right)^{s-1} M_{V}(2 s-1) .
$$

Various moments of $\gamma$ are computed from this by setting $s=p+1$. Using this expression for the average SNR computation, and by selecting scaling factors proportional to path-loss due to distance, the appropriate hop distance and relay amplification factor can be designed for a threshold average SNR for each compound distribution.

\subsubsection{Average SNR of $n$-Weibull channel}

The MT of $\Upsilon$ for $n$-Weibull distribution is given by Equation 8, with $s$ replaced by $2 s-1$ :

$$
M_{\Upsilon}(s)=\alpha^{(2 s-2) n} \Gamma^{n}\left(\frac{2 s-2+\beta}{\beta}\right)
$$

Using this, the expression for the $p$ th moment of SNR for $n$-Weibull distribution is

$$
m_{p}^{(\gamma)}=\alpha^{2 p n} \Gamma^{n}\left(\frac{\beta+2 p}{\beta}\right)\left(A^{2}\right)^{p} .
$$

Then, the average SNR for $n$-Weibull channel is computed as

$$
m_{1}^{(\gamma)}=\bar{\gamma}=\alpha^{2 n} \Gamma^{n}\left(\frac{\beta+2}{\beta}\right) A^{2} .
$$




\subsubsection{Average SNR of NW channel}

As in the previous case, the MT of the $p d f$ of $\Upsilon$ for NW channel is given by Equation 14 with $s$ replaced by $2 s-1$

$$
\begin{aligned}
M_{\Upsilon}(s)^{(\mathrm{NW})}= & \left(\frac{\Omega \alpha^{2}}{m}\right)^{-1} \frac{1}{\Gamma(m)} \Gamma(m+s-1) \\
& \times \Gamma\left(1-\frac{2}{\beta}+\frac{2 s}{\beta}\right)\left(\frac{\Omega \alpha^{2}}{m}\right)^{s},
\end{aligned}
$$

from which the $p$ th order moment of SNR is

$$
m_{p}^{\left(\gamma_{\mathrm{NW}}\right)}=\left(\frac{\Omega \alpha^{2}}{m}\right)^{p} \frac{1}{\Gamma(m)} \Gamma(m+p) \Gamma\left(1+\frac{2 p}{\beta}\right)\left(A^{2}\right)^{p} .
$$

Thus, the average SNR of the Nakagami $\times$ Weibull dualhop compound channel is

$$
m_{1}^{\left(\gamma_{\mathrm{NW}}\right)}=E[\gamma]=\left(\frac{\Omega \alpha^{2}}{m}\right) \frac{1}{\Gamma(m)} \Gamma(m+1) \Gamma\left(1+\frac{2}{\beta}\right) A^{2} .
$$

\subsubsection{Average SNR of RW channel}

Repeating the approach to the RW case, from Equation 20, the MT of the $p d f$ of $\Upsilon$ for RW case is given by

$$
M_{\Upsilon}(s)^{(\mathrm{RW})}=\alpha_{2}^{2 s-2} \Gamma(s) \Gamma\left(\frac{2 s+\beta-2}{\beta}\right)
$$

from which the $p$ th moment of SNR is

$$
m_{p}^{\left(\gamma_{\mathrm{RW}}\right)}=\alpha_{2}^{2 p} \Gamma(p+1) \Gamma\left(1+\frac{2 p}{\beta}\right)\left(A^{2}\right)^{p} .
$$

Hence, average SNR of the Rayleigh $\times$ Weibull compound channel is

$$
m_{1}^{\left(\gamma_{\mathrm{RW}}\right)}=\alpha_{2}^{2} \Gamma(2) \Gamma\left(1+\frac{2}{\beta}\right) A^{2} .
$$

In order to validate these theoretical expressions, simulation studies were conducted and the comparative results are plotted in Figure 5 for $\beta=m=4$. Based on Figure 5, for a two-hop transparent relay system, the required overall amplification factor for a target average SNR can be computed. For a system operating over NW channel, the required amplification factor will be more compared to that of RW or NW channel.

Comparison of Equations 46 and 40 reveals that the average SNR of RW compound channel reduces to that of a single-hop Weibull channel if $\lambda$ is unity, since $\Gamma(2)=1$, and $\lambda . \alpha=\alpha_{2}$. (With reference to Equation $4, \lambda=1$ corresponds to Rayleigh distribution with variance 0.5 .)

A single-stream two-hop AF relay transmission system was simulated for a relay amplification factor of 2 , to investigate the error performance of the system over each compound channel.

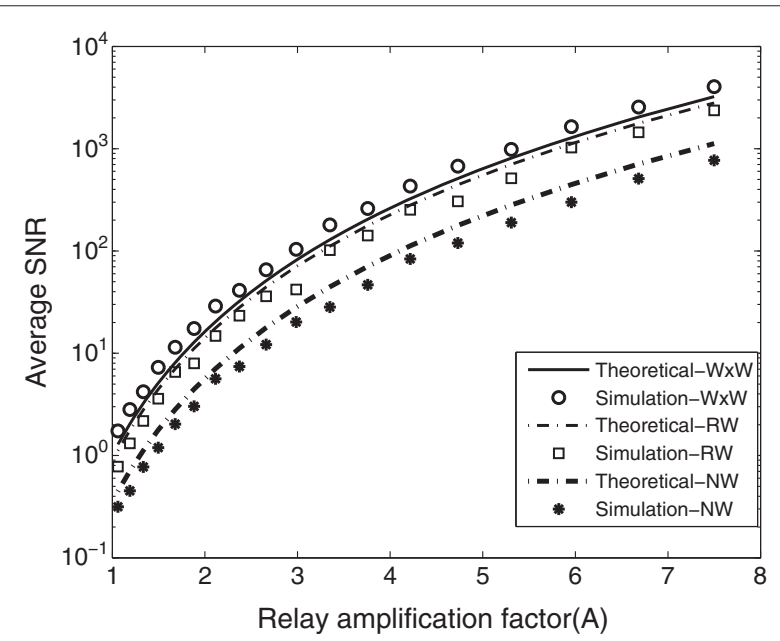

Figure 5 Average SNR of compound channels of Weibull $x$ Weibull(WW), Rayleigh $\times$ Weibull (RW), and Nakagami $x$ Weibull (NW) distributions. Average SNR from simulated samples shows close match to that evaluated from theoretical expressions at specific values of fading factors $\beta=m=4$. It is plotted against overall relay amplification factor. The required amplification factor for a target average SNR of the system operating under NW or RW or NW channel model can be obtained from this figure.

\subsection{Outage probability}

QoS of a system operating over fading channels can be predicted using the outage probability metric. It gives the probability that the instantaneous error rate exceeds a specified value. This metric, denoted by $P_{\text {out }}$, is equivalently defined as the the probability that the received SNR, $\gamma$, falls below a certain specified threshold, $\gamma_{\text {th }}$ :

$$
P_{\text {out }}=\int_{0}^{\gamma_{\text {th }}} f_{\gamma}(\gamma) d \gamma
$$

which is thecdf of $\gamma$, evaluated at $\gamma=\gamma_{\text {th }}$. To compute the outage, the $p d f$ of SNR is to be obtained first through the inverse MT approach.

\subsubsection{Outage probability for $n$-Weibull channel}

The SNR $p d f$ for $n$-Weibull compound channel is obtained by substituting Equation 38 in Equation 37, and finding the inverse MT:

$f_{\gamma}(\gamma)=\frac{\beta / 2}{2 c \pi j \alpha^{2 n}} \int_{L}\left[\Gamma\left(1-\frac{2}{\beta}+s^{\prime}\right)\right]^{n}\left(\frac{\gamma}{c \alpha^{n}}\right)^{-\beta / 2 s^{\prime}} d s^{\prime}$,

where $s^{\prime}=\frac{2 s}{\beta}$, and $c=\frac{E_{s}}{N_{0}}$. Rewriting this integral in terms of the Meijer G-function, it becomes

$$
f_{\gamma}(\gamma)=\frac{\beta}{2 c \alpha^{2 n}} G_{0, n}^{n, 0}\left(\left.z\right|_{1-\frac{2}{\beta}, 1-\frac{2}{\beta} \ldots} ^{-}\right),
$$

where $z=\gamma^{\beta / 2} c \alpha^{-n \beta / 2}$. Substituting Equation 48 in Equation 47, we get the outage probability for $n$-Weibull 
channel, which is a double integral. Using gamma reduction rule, the inner integral can be simplified as

$$
T_{2}=\int_{0}^{\gamma_{\mathrm{th}}} \gamma^{-\frac{\beta}{2} s^{\prime}} d \gamma=\frac{\gamma_{\mathrm{th}}^{-\frac{\beta}{2} s^{\prime}+1} \Gamma\left(\frac{2}{\beta}-s^{\prime}\right)}{\Gamma\left(1+\frac{2}{\beta}-s^{\prime}\right)} .
$$

Substituting this in to the outer integral, we get

$$
\begin{aligned}
P_{\text {out }}=F_{\gamma}\left(\gamma_{\text {th }}\right)= & \frac{c_{1}}{2 \pi j} \int_{L} \frac{\Gamma\left(\frac{2}{\beta}-s^{\prime}\right) \Gamma\left(1-\frac{2}{\beta}+s^{\prime}\right)^{n}}{\Gamma\left(1+\frac{2}{\beta}-s^{\prime}\right)} \\
& \times\left(\frac{\gamma_{\text {th }}^{\frac{\beta}{2}}}{\left(c \alpha^{n}\right)^{\beta / 2}}\right)^{s^{\prime}} d s^{\prime}
\end{aligned}
$$

where $c_{1}=c \frac{\beta}{2} \alpha^{-2 n}$. Denoting $z_{1}=\left(\gamma_{\mathrm{th}} c^{-1} \alpha^{-n}\right)^{\beta / 2}$ and expressing in terms of Meijer G-function, the outage probability for $n$-Weibull channel is

$$
P_{\text {out }}^{(\mathrm{W})}=c_{1} G_{1, n+1}^{n, 1}\left(\left.z_{1}\right|_{1-\frac{2}{\beta}, 1-\frac{2}{\beta} \ldots, \frac{-2}{\beta}} ^{1-\frac{2}{\beta}}\right),
$$

\subsubsection{Outage probability for NW channel}

Substituting Equation 41 in Equation 37 and finding the inverse MT, the $p d f$ of SNR for NW channel can be obtained as

$$
\begin{aligned}
f_{\mathrm{NW}}(\gamma)= & \frac{1}{2 D^{2} \Gamma(m) c} \frac{1}{2 \pi j} \int_{L} \Gamma(m+s-1) \Gamma \\
& \times\left(1-\frac{2}{\beta}+\frac{2 s}{\beta}\right)\left(c D^{2}\right)^{s} \gamma^{-s} d s,
\end{aligned}
$$

Changing the transform variable, for integer even values of $\beta$ greater than 2, this gets reduced to

$$
\begin{aligned}
f_{\mathrm{NW}}(\gamma)= & \frac{\beta}{2 N_{1}} \frac{1}{2 \pi j} \int_{L} \Gamma\left(m+\frac{\beta s^{\prime}}{2}-1\right) \Gamma \\
& \times\left(1-\frac{2}{\beta}+s^{\prime}\right)\left(c D^{2}\right)^{\frac{\beta s^{\prime}}{2}} \gamma^{\frac{-\beta s^{\prime}}{2}} d s^{\prime},
\end{aligned}
$$

where $N_{1}=D^{2} \Gamma(m) c$. To reduce this to a tractable form in Meijer G-function, apply gamma duplication formula to the arguments of first gamma function. This results in

$$
\begin{aligned}
f_{\mathrm{NW}}(\gamma)= & \frac{N_{2}}{2 N_{1} \pi j} \int_{L} \Pi_{j=0}^{b-1} \Gamma\left(\frac{m-1}{b}+\frac{j}{b}+s^{\prime}\right) \Gamma \\
& \times\left(1-\frac{2}{\beta}+s^{\prime}\right)\left(\frac{\gamma}{c D^{2}}\right)^{\frac{-\beta s^{\prime}}{2}} d s^{\prime},
\end{aligned}
$$

where $N_{2}=b^{m-1}(2 \pi)^{\frac{1-b}{2}}$. Substituting Equation 55 in Equation 47, and following the previous approach for simplification, we get $P_{\text {out }}$ as

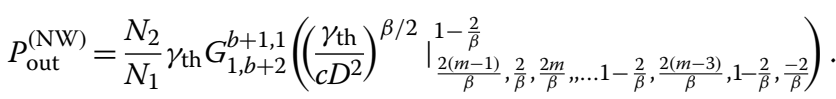

\subsubsection{Outage probability for RW channel}

Substituting Equation 44 in Equation 37 and taking the inverse MT, we get the $p d f$ of the corresponding SNR as

$$
\begin{aligned}
f_{\mathrm{RW}}(\gamma)= & \frac{\beta \alpha_{2}^{-} 2}{4 c \pi j} \int_{L} \Gamma\left(\frac{\beta}{2} s^{\prime}\right) \Gamma\left(1-\frac{2}{\beta}+s^{\prime}\right) \\
& \times\left(\frac{\gamma^{\frac{\beta}{2}}}{\left(c \alpha_{2}\right)^{\beta / 2}}\right)^{-s^{\prime}} d s^{\prime},
\end{aligned}
$$

for the variable transformation $s^{\prime}=\frac{2 s}{\beta}$. Again substituting this into Equation 47 and following the previous approach, $P_{\text {out }}$ is obtained in Meijer G-function as

$$
P_{\text {out }}^{(\mathrm{RW})}=c_{2} G_{1, \frac{\beta}{2}+2}^{\frac{\beta}{2}+1,1}\left(\left.z_{2}\right|_{0, \frac{2}{\beta}, \frac{4}{\beta}, \ldots 1-\frac{2}{\beta}, 1-\frac{2}{\beta}, \frac{2}{\beta}} ^{1-\frac{2}{\beta}}\right),
$$

where $c_{2}=c \frac{\beta}{2}^{\frac{1}{2}} 2 \pi^{1-\frac{\beta}{4}} \alpha_{2}^{2} \gamma_{\text {th }}$ and $z_{2}=\frac{\gamma_{\text {th }}^{\frac{\beta}{2}}}{\left(c b \alpha_{2}\right)^{\beta / 2}}$. Using these expressions, the exact probability that the received SNR is above a threshold can be computed by evaluating $1-P_{\text {out }}$ and is plotted in Figure 6.

\section{Simulation studies}

The derived expressions for $c d f$, $\mathrm{CV}$, and average SNR were validated by Montecarlo simulations. The various sample data corresponding to each basic distribution were generated, and the respective product channels, for both homogeneous ( $n$-Weibull) as well as heterogeneous (NW, RW) channels, were obtained. Weibull distributed coefficients $W_{i}$ were generated using the Gaussian in-phase components $W_{i 1}$ and quadrature components $W_{i 2}$ as in [21]:

$$
W_{i}=\left(W_{i 1}+j W_{i 2}\right)^{2 / \beta},
$$

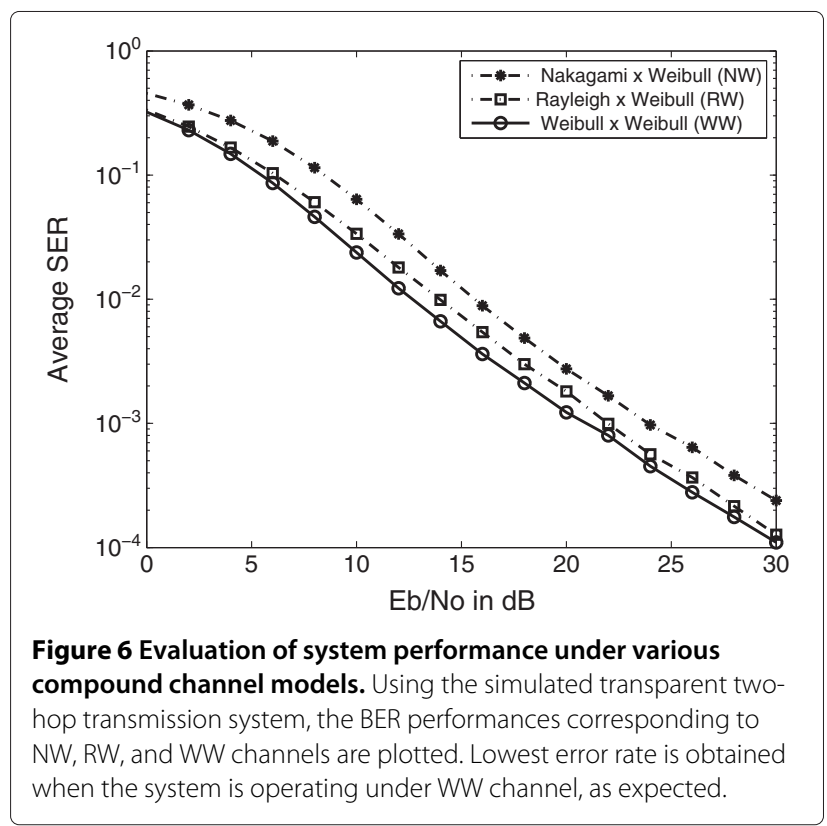


Table 1 Comparison of analytical $c d f$ to simulation $c d f$

\begin{tabular}{lccccccc}
\hline Product distributions & \multicolumn{2}{c}{ NW } & \multicolumn{2}{c}{ RW } & \multicolumn{2}{c}{ WW } \\
\hline Threshold $\left(\boldsymbol{v}_{\boldsymbol{t}}\right)$ & Empirical $\boldsymbol{c d f}$ & Analytical $\boldsymbol{c d f}$ & Empirical $\boldsymbol{c d f}$ & Analytical $\boldsymbol{c d f}$ & Empirical $\boldsymbol{c d f}$ & Analytical $\boldsymbol{c d f}$ \\
\hline 0.5 & 0.0823 & 0.0779 & 0.4266 & 0.4478 & 0.4508 & 0.4609 \\
\hline 0.7 & 0.1322 & 0.1217 & 0.4677 & 0.4782 & 0.5066 & 0.5174 \\
\hline 0.9 & 0.1245 & 0.1348 & 0.5201 & 0.5217 & 0.5577 & 0.5609 \\
\hline 1 & 0.3256 & 0.3217 & 0.6789 & 0.6869 & 0.7567 & 0.7695 \\
\hline 1.2 & 0.4912 & 0.4956 & 0.7800 & 0.7862 & 0.8701 & 0.8764 \\
\hline
\end{tabular}

where $j=\sqrt{-1}$. The Rayleigh channel coefficients were generated in the same way with $\beta=2$, and the Nakagami$m$ using the Gamma random variates from the Matlab software package. Using these random variates generated for the different constituent channel coefficients with various parameter values, the corresponding product channel coefficients were obtained for different hop count values.

For the analytical and simulation $c d f$ comparison in Table 1, data samples were generated with $m=\beta=4$ and unity scaling factors for the various given distributions.

The empirical $c d f$ was computed from the generated samples $\left\{v_{1}, v_{2}, \ldots . v_{z}\right\}$ as:

$$
F(v)=\frac{1}{Z} \sum_{i=1}^{Z} 1_{\left\{v_{i} \leq v_{t}\right\}},
$$

where $1_{\left\{v_{i} \leq v_{t}\right\}}$ represents an indicator function which takes value unity when $v_{i} \leq v_{t}$, and $Z$ represents the total number of observations.

For the computation of empirical CV, sample moments are to be evaluated. From the generated data, the sample moments were computed as

$$
\begin{aligned}
& m_{1}=\frac{1}{Z} \sum_{i=1}^{Z} v_{i} \\
& m_{2}=\frac{1}{Z} \sum_{i=1}^{Z} v_{i}^{2}
\end{aligned}
$$

The empirical CV was then computed from the sample moments and using the basic definition of $\mathrm{CV}$ :

$$
\mathrm{CV}_{\mathrm{e}}=\sqrt{\frac{m_{2}}{m_{1}^{2}}-1}
$$

The empirical $\mathrm{CV}$, denoted as $\mathrm{CV}_{e}$, was computed from the samples generated for various values of $\beta, m$ and $n$. For the computation of the sample moments and $\mathrm{CV}_{e}, 10^{6}$ samples were generated in each of the homogeneous and heterogeneous compound channel cases. The exact match between the theoretical CV and Empirical CV shows the accuracy of the exact expressions derived for $\mathrm{CV}$ and moments.
In order to validate the expressions for average SNR, different sample data were generated with $m=\beta=4$. The scaling factors for the samples were selected as relay amplification factor. A single-stream two-hop AF relay transmission system was also simulated for a relay amplification factor of 2, to analyse the error performance of the system over each compound channel. BPSK modulated signals were transmitted, and additive white Gaussian noise was added at each hop. Corresponding to NW distributions, the first hop was modeled by Nakagami- $m$ with $m=4$ and the second hop was modeled by Weibull with $\beta=4$ distributed coefficients. Scale factors were selected as unity for both distributions, so that relay amplification factor stands for the scale factor for the samples, when implemented to the system. Similarly, RW and WW compound channel models were also developed. The received signals were detected using zero crossing detector. Average bit error rate (BER) performance of the system corresponding to the three channel models was thus evaluated using simulations and plotted in Figure 7. The sample data generated were also used to obtain an empirical q-q plot of the quantiles of dataset-1 (WW) versus the quantiles of the dataset-2 (NW or RW).

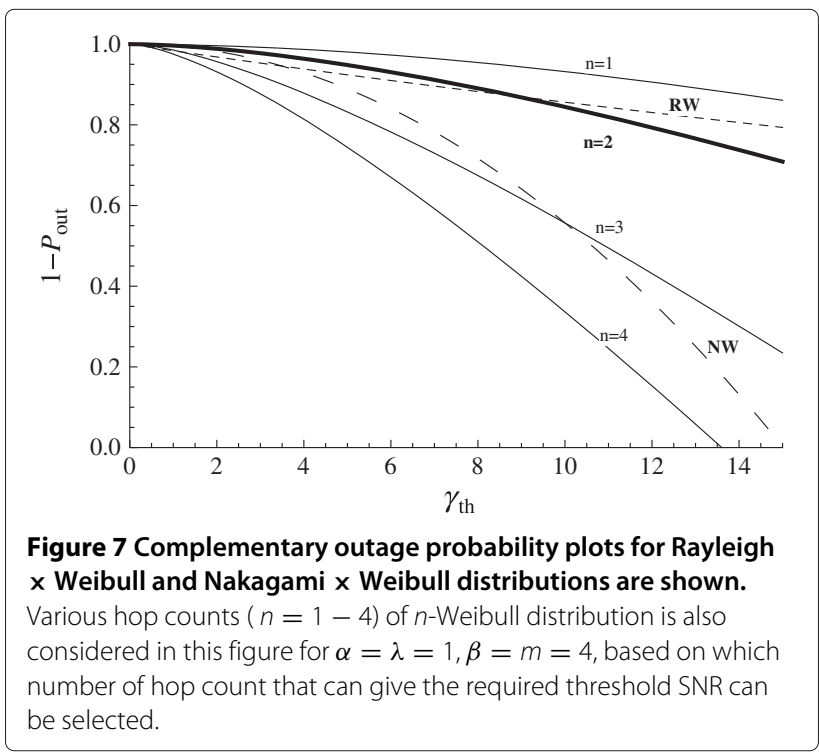




\section{Results and discussions}

The analytical and simulation results obtained are discussed in this section. The plots of derived analytical $p d f$ for Weibull $\times$ Weibull $(W W)$, Nakagami $\times$ Weibull $(N W)$, and Rayleigh $\times$ Weibull $(\mathrm{RW})$ are shown in Figure 2. It may be noted that the $p d f$ of WW distribution approaches Gaussian distribution for $\beta=4$. These exact $p d f$ expressions can be used to identify the distribution from empirical data, using q-q plots.

Figure 3 shows the plots corresponding to the derived exact expressions of $c d f$ for different values of $n$ and for the NW, RW cases. Knowledge of $c d f$ can be used to fix the dynamic range (DR) at the receiver unit.

The DR can be obtained as the ratio $v_{t \max } / v_{t \min }$ and the thresholds are decided based on the $c d f$ values. It is seen from Figure 3 that the required DR at receiver will have to be increased as the order of multiplication increases. The smaller slope of $c d f$ for RW and NW cases, compared to the 2-Weibull (WW) case, and the corresponding difference in DR are obvious from Figure 3. Simulation results show exact match to the analytical results as tabulated in Table 1.

Lower values of $\mathrm{CV}$ indicate better regularity of the channel coefficients. In Figure 4, CV is plotted against fading factors $\beta$ or $m$. For $\beta=2$, the RW and WW distributions give the same $\mathrm{CV}$, as both distributions reduce to RR (two-Rayleigh) distribution. Among the three distributions, NW shows the minimum variability (most regular), as expected. Simulation and analytical values $\left(C V_{e}, \mathrm{CV}\right)$ show exact match in Figure 4, proving the accuracy of the derived analytical expressions. The exact match between the theoretical CV and Empirical CV shows not only the accuracy of the expressions derived for $\mathrm{CV}$ but also the exact agreement of the derived (population) moments to the sample moments.

The average SNR computed analytically and through simulation (as discussed in Section 6) for each case are plotted against the relay amplification factor in Figure 5. The close match between the simulation and theoretical plots observed in Figure 5 shows the accuracy of the derived expressions for average SNR of WW, RW, and NW channel models. The lowest average SNR for the NW channel under the given fading factors $(\beta=m=1)$ means that the required amplification factor for a target average SNR for a system operating over this channel will be higher compared to that of RW or NW channels. Based on Figure 5, the required overall amplification factor for any target average SNR can be computed.

The plot of $1-P_{\text {out }}$ in Figure 6 gives the probability of the received SNR to be above a threshold, for $n$-Weibull compound channel with various hop counts. Based on the minimum $\gamma_{\text {th }}$ required at the receiver, the number of hop counts can be selected. Also shown in Figure 6 are the plots for the NW and RW dual-hop compound channels. The improved performance of RW case over 2Weibull case, in the high SNR region, and the degradation of NW channel over the homogeneous case are exposed in Figure 6.

In order to demonstrate the application of the derived average SNR, a two-hop transparent relay transmission system was simulated as discussed in Section 6. The average BER performance of the system over NW, RW, and WW channels are plotted in Figure 7. Obviously, maximum error rate occurs with NW channel as it delivers minimum average SNR. For the system operating over $\mathrm{NW}$ channel, an additional $3-\mathrm{dB}$ increment in $\frac{E_{b}}{N_{0}}$ is required for achieving an average BER of $10^{-3}$ in comparison with WW channel scenario.

The quantile-quantile (q-q) plot in Figure 8 shows the quantile deviations of the models NW, RW with respect to a homogeneous model WW. The first and third quartiles of each dataset are found first, which are extrapolated out to the ends of the samples to evaluate the linearity of the data. These are shown as the reference plots 1 to 3 . The departure from these reference lines indicates that the two data sets have come from populations with different distributions. The horizontal axis corresponds to estimated quantiles from data set-1 (WW), while vertical axis corresponds to estimated quantiles from data set2 (RW or NW). Even though the distributions, Rayleigh and Weibull, belong to the same class of distributions, the quantiles of RW are different from that of WW.

From the derived exact expressions for the various primary statistics and the corresponding simulation results, it is evident that the end-to-end channel distribution

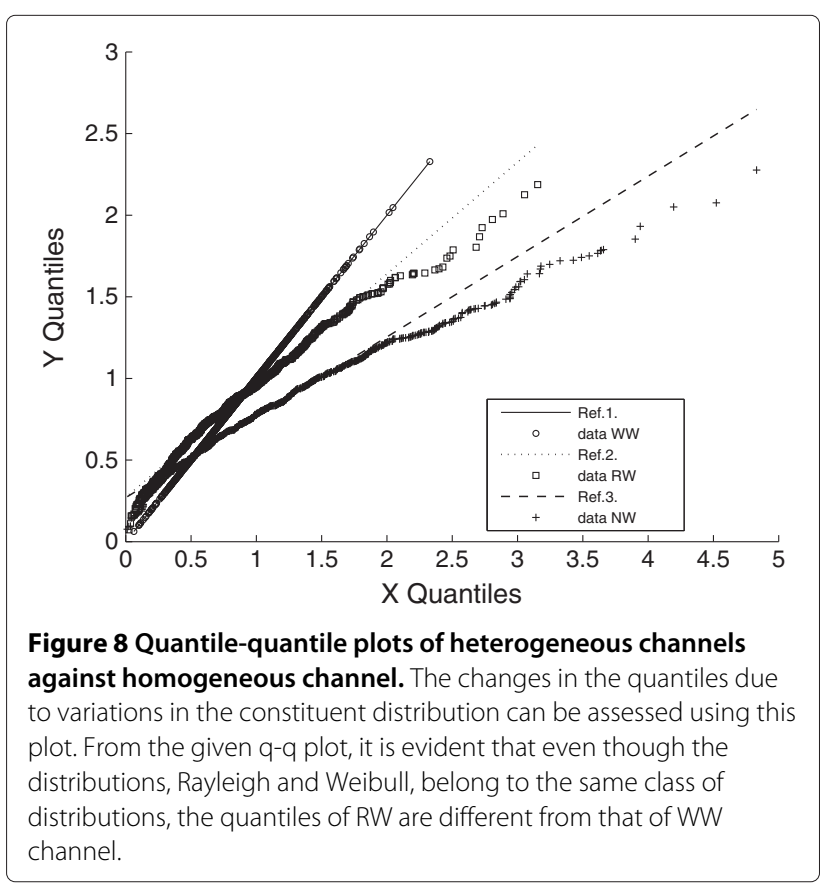


for different number of hops may deviate significantly in modality, skewness, Kurtosis, etc., due to cascading. The derived performance metrics depend on the number of hops and the constituent distributions. These exact expressions, and the unified approach that can be used for other cases as well, are expected to be of great use in the realistic system design and performance evaluation of the multi-hop, diversity attaining transparent cooperative systems.

\section{Conclusion}

Exact expressions are derived for the moments, $p d f$, and $c d f$, of the proposed heterogeneous compound channel models in multi-hop transparent cooperative relay transmissions for outdoor applications. The statistical properties of heterogeneous compound channels, Nakagami $\times$ Weibull and Rayleigh $\times$ Weibull, are compared with that of homogeneous compound channel assumption. The expressions are derived in tractable compact forms, in terms of Meijer G-functions, so that accurate and easy computations are possible. Coefficient of variation, average SNR, and outage probability are the performance metrics derived to quantify the variability, fidelity, and acceptable SNR level of the system, respectively. Corresponding to the SNR statistics, a two-hop transparent relay transmission system is simulated, and the average BER performance of the system under the proposed channel models are also evaluated. The approach can be easily extended to other combinations of distributions also, if the constituent channels are Mellin transform defined. The results are expected to be useful in the design of receivers in multi-hop diversity attaining cooperative relay systems, to achieve predefined QoS.

\section{Appendix}

\section{A.1 General formulae used Meijer G-function:}

The general Meijer G-function, defined using the contour integral equation of ratios of products of Gamma functions, is given by:

$$
G_{p, q}^{m, n}\left(\left.z\right|_{b_{1}, \ldots b_{q}} ^{a_{1}, \ldots a_{p}}\right)=\frac{1}{2 \pi j} \int_{L} \frac{\prod_{i=1}^{m} \Gamma\left(b_{i}+s\right) \prod_{i=1}^{n} \Gamma\left(1-a_{i}-s\right)}{\prod_{i=n+1}^{p} \Gamma\left(a_{i}+s\right) \prod_{i=1+m}^{q} \Gamma\left(1-b_{i}-r\right)} z^{-s} d s,
$$

where $\Gamma($.$) is the complete gamma function [22]$ [Eqn.9.301].

\section{Gamma duplication formula[19]:}

$$
\Gamma(b x)=(2 \pi)^{\frac{1-b}{2}} b^{b x-\frac{1}{2} \prod_{i=0}^{b-1} \Gamma\left(\frac{i}{b}+x\right)}
$$

\section{Gamma reduction rule:}

$$
\Gamma(x+1)=x \Gamma(x)
$$

\section{A.2 Analytical Steps for the Integral Evaluations}

The inverse integral equations need to be reduced into a computable Meijer G-function format. As software packages do not have built-in subroutines for the evaluation of such integrals for heterogeneous combinations of distributions, appropriate format conversion is necessary. For illustration, details of the conversion of $p d f$ of NW channel and $c d f$ of RW channel are given below.

\section{A.2.1 Steps for the computation of pdf for NW case:}

Considering the MT of NW channel given by the Equation 14, the coefficient of the transform variable $s$ in the two arguments of gamma functions are neither identical nor unity (in most of the cases, this will not be unity). Transformation of one of the coefficients to unity will alter the other coefficient. Hence, gamma duplication formulae can be used at this stage to convert the variable coefficient of the first argument to unity. Thus, the $p d f$ of NW channel given by Equation 14 is converted as

$$
\begin{aligned}
f_{\mathrm{NW}}(v)= & \frac{\beta}{\Gamma(m)}\left(\frac{\Omega \alpha}{m}\right)^{\frac{1}{2}} \frac{1}{2 \pi j} \int_{L} \Gamma\left(m-\frac{1}{2}+\frac{\beta s^{\prime}}{2}\right) \Gamma \\
& \times\left(1-\frac{1}{\beta}+s^{\prime}\right)\left(\frac{\Omega \alpha^{2}}{m}\right)^{\frac{s^{\prime} \beta}{2}} v^{-\beta s^{\prime}} d s^{\prime},
\end{aligned}
$$

where $s^{\prime}=\frac{s}{\beta}$. Now, the first gamma function term contains a transform variable $s^{\prime}$ with coefficient as $\frac{\beta}{2}$. This can be eliminated by the application of appropriate gamma duplication formula, which will reduce the expression to a more convenient form as

$$
\begin{aligned}
& f_{\mathrm{NW}}(v)=\frac{\beta}{\Gamma(m)}\left(\frac{\Omega \alpha}{m}\right)^{\frac{-1}{2}} \frac{1}{2 \pi j} \int_{L}(2 \pi)^{\frac{1-\beta / 2}{2}} \frac{\beta}{2}^{\frac{\beta}{2}\left(\frac{2 m-1}{\beta}+s^{\prime}\right)} \prod_{i=0}^{\frac{\beta}{2}-1} \Gamma\left(\frac{2 m-1}{\beta}+s^{\prime}+\frac{i}{\beta / 2}\right) \\
& \Gamma\left(1-\frac{1}{\beta}+s^{\prime}\right)\left(\frac{v^{\beta}}{\left(\frac{\Omega \alpha}{m}\right)^{\frac{\beta}{2}}}\right)^{-s^{\prime}} d s^{\prime}
\end{aligned}
$$


this can be expanded as

$$
\begin{aligned}
f_{\mathrm{NW}}(v)= & (2 \pi)^{\frac{1-b}{2}} \frac{2(\beta / 2)^{m}}{\Gamma(m)}\left(\frac{\Omega \alpha}{m}\right)^{\frac{-1}{2}} \frac{1}{2 \pi j} \int_{L} \Gamma\left(\frac{2 m-1}{b}+s^{\prime}\right) \\
& \Gamma\left(\frac{2 m-1}{b}+\frac{1}{b}+s^{\prime}\right) \\
& \Gamma\left(\frac{2 m-1}{b}+\frac{b-1}{b}+s^{\prime}\right) \ldots . . \Gamma\left(1-\frac{1}{2 b}+s^{\prime}\right) \\
& \times\left(\frac{v^{\beta}}{\left(b \frac{\Omega \alpha}{m}\right)^{\frac{\beta}{2}}}\right)^{-s^{\prime}} d s^{\prime} .
\end{aligned}
$$

Now, the expression can be converted to general Meijer-G format (Equation 64), and the corresponding computable $p d f$ expression is obtained as

$$
f_{\mathrm{NW}}(v)=\frac{K \beta b^{b}}{D \Gamma(m)} G_{0, b}^{b+1,0}\left(\left.\frac{v^{\beta}}{\left(b^{\frac{1}{2}} D\right)^{\beta}}\right|_{\frac{2 m-1}{\beta}, \frac{2 m+1}{\beta}, \ldots, \frac{2 m+\beta-3}{\beta}, 1-\frac{1}{\beta}} ^{-}\right),
$$

where $b=\frac{\beta}{2}, D=\left(\frac{\Omega \alpha^{2}}{m}\right)^{\frac{1}{2}}$, and $K=\frac{\sqrt{2 \pi}}{2 \pi^{b / 2}}$. For computing the $p d f$ of NW channel at $m=\beta \stackrel{2 \pi}{=} 4$ and unity scale factors, this function reduces to

$$
f_{\mathrm{NW}}(v)=\left(\frac{32}{\sqrt{(2 \pi)} \Gamma(4)}\right) G_{0,2}^{3,0}\left(\left.\frac{v^{4}}{2}\right|_{\frac{7}{4}, \frac{9}{4}, \frac{3}{4}} ^{-}\right) .
$$

Similarly, for RW channel, the computable $p d f$ at the same parameter values becomes

$$
f_{\mathrm{RW}}(v)=\frac{4}{\sqrt{2 \pi}} G_{0,2}^{3,0}\left(\left.\frac{v^{4}}{2}\right|_{\frac{3}{4}, \frac{1}{4}, \frac{3}{4}}\right) .
$$

\section{A.2.2 Steps for the computation of cdf for RW case:}

Integrating the $p d f$, given by Equation 23, with respect to $v$ and using gamma reduction rule (66), the $c d f$ for RW channel becomes

$$
\begin{aligned}
F_{\mathrm{RW}}\left(v_{t}\right)= & \left(\frac{\beta}{\alpha_{2}}\right) \frac{1}{2 \pi j} \int_{L} \Gamma\left(1-\frac{1}{\beta}+s^{\prime}\right) \Gamma\left(\frac{1}{2}+\frac{\beta s^{\prime}}{2}\right) \frac{\Gamma\left(\frac{1}{\beta}-s\right)}{\Gamma\left(1+\frac{1}{\beta}-s\right)} \\
& \times\left(\frac{v_{t}^{\beta}}{\alpha_{2}^{\beta}}\right)^{-s^{\prime}} d s^{\prime} .
\end{aligned}
$$

The next step is to convert this integral, using gamma duplication formula (65), in to the form given by:

$$
\begin{aligned}
F_{\mathrm{RW}}\left(v_{t}\right)= & \left(\frac{\beta}{\alpha_{2}}\right) \frac{\sqrt{2 \pi}}{(2 \pi)^{\frac{\beta}{4}}} \frac{1}{2 \pi j} \int_{L} \Gamma\left(1-\frac{1}{\beta}+s^{\prime}\right) \prod_{i=0}^{\frac{\beta}{2}-1} \\
& \times \Gamma\left(\frac{1}{\beta}+\frac{2 i}{\beta}+s^{\prime}\right) \frac{\Gamma\left(\frac{1}{\beta}-s\right)}{\Gamma\left(1+\frac{1}{\beta}-s\right)}\left(\frac{v_{t}^{\beta / 2}}{\alpha_{2}^{\beta / 2}}\right)^{-s^{\prime}} d s^{\prime}
\end{aligned}
$$

$$
\begin{aligned}
= & \left(\frac{\beta}{\alpha_{2}}\right) \frac{\sqrt{2 \pi}}{(2 \pi)^{\frac{\beta}{4}}} \frac{1}{2 \pi j} \int_{L} \Gamma\left(1-\frac{1}{\beta}+s^{\prime}\right) \Gamma\left(\frac{1}{\beta}+s^{\prime}\right) \Gamma\left(\frac{3}{\beta}+s^{\prime}\right) \ldots . \\
& \times \Gamma\left(1-\frac{1}{\beta}+s^{\prime}\right) \frac{\Gamma\left(\frac{1}{\beta}-s\right)}{\Gamma\left(1+\frac{1}{\beta}-s\right)}\left(\frac{v_{t}^{\beta / 2}}{\alpha_{2}^{\beta / 2}}\right)^{-s^{\prime}} d s^{\prime} .
\end{aligned}
$$

Converting this to Meijer-G format, the $c d f$ becomes

$$
F_{\mathrm{RW}}\left(v_{t}\right)=\frac{v_{t}}{\alpha_{2}} K G_{1, \beta / 2}^{\beta / 2+1,1}\left(\left.\frac{v_{t}^{\beta}}{\alpha_{2}^{\beta} b}\right|_{1-\frac{1}{\beta}, \frac{1}{\beta}, \frac{3}{\beta} \ldots 1-\frac{1}{\beta}, \frac{-1}{\beta}} ^{1-\frac{1}{\beta}}\right) .
$$

For specific parameter values as in 70 given above, the expression get reduced to the form:

$$
F_{\mathrm{RW}}\left(v_{t}\right)=\frac{v_{t}}{(2 \pi)^{1 / 2}} G_{1,2}^{3,1}\left(\left.\frac{v_{t}^{4}}{2}\right|_{\frac{3}{4}, \frac{1}{4}, \frac{3}{4}, \frac{-1}{4}} ^{\frac{3}{4}}\right) .
$$

Similarly for NW channel, the tractable $c d f$ becomes

$$
F_{\mathrm{NW}}\left(v_{1}\right)=\frac{16 v_{1}}{\Gamma(4) \sqrt{(2 \pi)}} G_{1,2}^{3,1}\left(\left.\left(2 v_{1}\right)^{4}\right|_{\frac{7}{4}, \frac{9}{4}, \frac{3}{4}, \frac{-1}{4}} ^{\frac{3}{4}}\right) .
$$

Adopting similar steps for the integral equations of SNR will facilitate the computations of SNR statistics also.

\section{Competing interests}

The authors declare that they have no competing interests.

Received: 28 June 2013 Accepted: 19 November 2013 Published: 5 December 2013

\section{References}

1. GK Karagiannidis, TA Tsiftsis, RK Mallik, Bounds for multihop relayed communications in Nakagami-m fading. Commun. IEEE Trans. 54(1), 18-22 (2006)

2. NC Sagias, GS Tombras, On the cascaded Weibull fading channel model. J. Franklin Inst. 344(1), 1-11 (2007)

3. KK Cibile, SM Sameer, L Jacob, On the computation of exact moments and performance metrics for multi-hop transparent Weibull relay channels. National conf. on Commun. NCC-2013, New Delhi, 1-5 February 2013

4. D Chizhik, G Foschini, M Gans, R Valenzuela, Keyholes, correlations, and capacities of multielement transmit and receive antennas. Wireless Commun. IEEE Trans. 1(2), 361-368 (2002)

5. Y Chen, GK Karagiannidis, H Lu, N Cao, Novel approximations to the statistics of products of independent random variables and their applications in wireless communications. Vehicular Technol. IEEE Trans. 61(2), 443-454 (2012)

6. GK Karagiannidis, Performance bounds of multihop wireless communications with blind relays over generalized fading channels. Wireless Commun. IEEE Trans. 5(3), 498-503 (2006)

7. J Salo, HM El-Sallabi, P Vainikainen, The distribution of the product of independent rayleigh random variables. Antennas Propagation IEEE Trans. 54(2), 639-643 (2006)

8. GK Karagiannidis, DA Zogas, NC Sagias, TA Tsiftsis, PT Mathiopoulos, Multihop communications with fixed gain relays over generalized fading channels. GLOBECOM'04 IEEE, Dallas, December 2004

9. MJ David, FP Jose, KK Wong, Closed-form analysis of multibranch switched diversity with noncoherent and differentially coherent detection. Int. J. Commun. Syst. 5(26), 127-13 (2013)

10. TSB Reddy, R Subadar, P Sahu, Outage probability of SC receiver over exponentially correlated k fading channels. Commun. Lett. IEEE. 14(2), 118-120 (2010)

11. FSAl-Qahtani, C Zhong, KA Qaraqe, AH Inuweiri, T Ratnarajah, Performance analysis of fixed-gain AF dual-hop relaying systems over 
Nakagami-m fading channels in the presence of interference. EURASIP J. Wireless Commun. Netw. 2011(1), 1-10 (2011)

12. CK Datsikas, KP Peppas, NC Sagias, GS Tombras, Serial relaying communications over generalized-gamma fading channels. Wireless Commun. Mobile Comput. 12(13), 1191-1202 (2012)

13. G Karagiannidis, NC Sagias, PT Mathiopoulos, N-Nakagami a novel stochastic model for cascaded fading channels. Commun. IEEE Trans. 55(8), 1453-1458 (2007)

14. F Yilmaz, M-S Alouini, Product of the powers of generalized Nakagami-m variates and performance of cascaded fading channels. GLOBECOM 2009 IEEE, USA, December 2009

15. J Malhotra, Performance of multi-hop communication with fixed-gain relays over Weibull fading channels. IJAST. 2(4), 1-8 (2011)

16. SS Ikki, MH Ahmed, Performance analysis of dual hop relaying over non-identical Weibull fading channels. VTC 2009, Barcelona, April 2009

17. S Majhi, Y Nasser, JF Hélard, Outage probability analysis of cooperative communications over asymmetric fading channel, in communication and networking. ed. by J Peng, ( ISBN 978-953-307-114-5, Sciyo, Croatia 2010) p. 434

18. KK Cibile, SM Sameer, L Jacob, A robust channel equaliser with noise correlation subtraction for AF relay cooperative networks. Annual IEEE Intl. Conf. INDICON-2012, Kochi, 7-9 (December 2012)

19. S Khairnar, R Pise, J Salunkhe, Study of the mellin integral transform with applications in statistics and probability. Arch. Appl. Sci. Res. 4(3), 1294-1310 (2012)

20. MK Simon, M-S Alouini, Digital Communication over Fading Channels. (John Wiley, New York, 2005)

21. S Nallagonda, S Roy, S Kundu, Performance of cooperative spectrum sensing in rician and Weibull fading channels. Annual IEEE Intl. Conf. INDICON-2011, Hyderabad, 16-18 (December 2011)

22. IL Gradshteyn, I Ryzhik, Errata for Table of Integrals, Series, and Products. (Academic Press, Orlando, 2005)

\section{doi:10.1186/1687-1499-2013-274}

Cite this article as: Kanjirathumkal et al: A unified approach for the fading statistics of heterogeneous compound channels in transparent relay transmissions. EURASIP Journal on Wireless Communications and Networking 2013 2013:274

\section{Submit your manuscript to a SpringerOpen ${ }^{\circ}$ journal and benefit from:}

- Convenient online submission

- Rigorous peer review

- Immediate publication on acceptance

- Open access: articles freely available online

- High visibility within the field

- Retaining the copyright to your article

Submit your next manuscript at $\boldsymbol{\wedge}$ springeropen.com 